\title{
CPT-TODIM METHOD FOR INTERVAL NEUTROSOPHIC MAGDM AND ITS APPLICATION TO THIRD-PARTY LOGISTICS SERVICE PROVIDERS SELECTION
}

\author{
Dan ZHANG ${ }^{1}$, Yong $\mathrm{SU}^{2}$, Mengwei $\mathrm{ZHAO}^{2}$, Xudong $\mathrm{CHEN}^{3^{*}}$ \\ ${ }^{1}$ Sichuan University Jinjiang College, 620860 Meishan, P.R. China \\ ${ }^{2}$ School of Business, Sichuan Normal University, 610101 Chengdu, P.R. China \\ ${ }^{3}$ School of Accounting, Southwestern University of Finance and Economics, \\ 611130 Chengdu, P.R. China
}

Received 08 April 2021; accepted 09 August 2021; first published online 19 November 2021

\begin{abstract}
The multiple attribute group decision making (MAGDM) has always been a concern in the research field. In this article, we establish the interval neutrosophic TODIM method based on cumulative prospect theory (CPT-IN-TODIM) for MAGDM issue. This new developed CPTIN-TODIM method has markedly superiority in describing decision maker's psychological states, which utilizes the weight function to adjust weighting attributes distinguishing from the classical TODIM method. Then, this new developed method has been applied to select the third-party logistics service providers and been expound on the disparity with existing methods. Finally, the results of contrastive analysis indicate that this new developed method can lead to the appropriate conclusion and sticks out the differences between alternatives to provide clearer direction. Hence, the new developed CPT-IN-TODIM method is reliable and valid.
\end{abstract}

Keywords: multiple attribute group decision making (MAGDM), interval neutrosophic sets, TODIM, cumulative prospect theory, third-party logistics service providers.

JEL Classification: C43, C61, D81.

\section{Introduction}

Both multiple attribute decision making (MADM) and multiple attribute group decision making (MAGDM) (Kim et al., 1999; Liao et al., 2021; Xu \& Chen, 2007; Zhao et al., 2021b) refer to the process of selecting the optimal alternative among several alternatives, and these alternatives involve multiple identical attributes (Chen et al., 2012; Lei et al., 2021; Wei et al., 2021). In our daily life, we have to face many complex and uncertain questions that cannot be answered with full determined or absolute negation, so does the management decisionmaking process. The uncertainty of environment makes it difficult to describe the attribute

*Corresponding author. E-mail: xutung99@126.com

Copyright $\odot 2021$ The Author(s). Published by Vilnius Gediminas Technical University

This is an Open Access article distributed under the terms of the Creative Commons Attribution License (http://creativecommons. org/licenses/by/4.0/), which permits unrestricted use, distribution, and reproduction in any medium, provided the original author and source are credited. 
value accurately with traditional mathematics. Therefore, Zadeh (1965) proposed the fuzzy set for the first time. Then, neutrosophic set created by Smarandache (2002) and consisted of the truth-membership, the indeterminacy-membership and the falsity-membership which were the standard or non-standard real subsets of ]-0,1+[. Wang et al. (2010) defined single-valued neutrosophic set (SVNS). Wang et al. (2005) designed the interval neutrosophic set (INS). Many scholars used SVNS or INS to study the problem of MADM or MAGDM (Biswas et al., 2016; Kahraman, et al., 2020; Meng et al., 2019; Nafei et al., 2019; Wu et al., 2018; Yang et al., 2020). Wang et al. (2019) researched the choice about strategic supplier by utilizing interval neutrosophic information. A series of aggregation operators about SVNS and INS are put forward by Garg and Nancy (2020) and in their mind, both objective factor and subjective factor are considered concurrently. Wang (2020) proposed a new interval neutrosophic MADM method taking example by the projection model. Awang et al. (2020) proposed the interval neutrosophic Shapley normalized weighted BM operator. Fan et al. (2021) developed interval-valued neutrosophic TODIM method for risk assessment. Pramanik et al. (2017) constructed neutrosophic cubic TODIM method. Pramanik et al. (2018) also considered to establish the VIKOR model under neutrosophic cubic environment. Pramanik et al. (2016) created bipolar neutrosophic TODIM method. Pramanik and Mallick (2019) investigated the feasibility of building TODIM model with trapezoidal neutrosophic number.

On the basis of the prospect theory (Kahneman \& Tversky, 1979), Gomes and Lima (1979) first established the TODIM method, which is effective to solve the MADM problems where the DMs' psychological behaviors are considered. The TODIM method had been widely applied in many fields, such as selection of green supplier (Nie et al., 2019), products selection (Liu \& Teng, 2019), Water security evaluation (Zhang et al., 2019). Moreover, many scholars combined TODIM method under different fuzzy sets. For example, Liang et al. (2019) built TODIM method under proportional hesitant fuzzy linguistic and environment. Liu et al. (2019) focused on fermatean fuzzy linguistic information. Ji et al. (2020) selected dual hesitant PFSs to investigate TODIM method.

Flavio and GonzIlez (2012) initially proposed the idea of the combination of TODIM and cumulative prospect theory (CPT). Tian et al. (2019) realized a more detailed improvement of TODIM by using CPT. Huang et al. (2021) defined EDAS method based on Prospect Theory. Zhao et al. (2021c) rebuilt the CPT-TODIM by using intuitionistic fuzzy information. Su et al. (2021) built the PT-TODIM method for probabilistic linguistic MAGDM. Zhao et al. (2021a) defined the Intuitionistic fuzzy MABAC method based on CPT. Zhao et al. (2021d) established interval-valued intuitionistic fuzzy TODIM model on the basis of CPT for studying the urban ecological risk. To a certain extent, increasing the risk weighting moderately is conducive to the enterprise's risk avoidance and conforms to the enterprise's decision-making requirements. Therefore, in my opinion, the CPT-TODIM method has obvious advantages in dealing with MAGDM problems. The TODIM method took into account decision makers' different psychological perceptions of gains and losses, but unfortunately it is only appropriate for certain types of decision makers. But the CPT-TODIM method can depict the difference of psychological perception for different types of decision makers. In addition, compared with other fuzzy sets, interval neutrosophic set is a more flexible and more applicable method for evaluating uncertain information. However, there are few studies 
using this method. Therefore, this paper aims to establish the interval neutrosophic TODIM based on cumulative prospect theory (CPT-IN-TODIM) method for selecting the cold chain logistics service providers. In addition, the new developed method also takes a more scientific approach, the entropy weight method, to acquire the original attribute weights avoiding the interference of subjective factors. Although other methods have been proposed to determine the weight of attributes (Keshavarz-Ghorabaee et al., 2019), entropy weight method is still a very effective and straightforward method.

The structure of such paper is as follows. In second part, we introduce the knowledge involved in this paper, such as the definition, the score and accuracy function of INSs, and CPT-TODIM method. After that, we demonstrate the developed interval neutrosophic TODIM method based on cumulative prospect theory more detail in third part. And then, in section 4 and 5, we not only apply this new developed method to select the third-party logistics service providers, but also expound on the disparity with existing methods. Finally, the results of contrastive analysis indicates that this new developed method can lead to the appropriate conclusion and sticks out the differences between alternatives to provide clearer direction.

\section{Preliminary knowledge}

In this section, we sort out the basic knowledge about INSs.

Definition 1 (Wang et al., 2010). A NS $Y$, which consists of the truth-membership $\rho_{Y}(n)$, indeterminacy-membership $\sigma_{Y}(n)$ and falsity-membership $\lambda_{Y}(n)$, can be expressed as follows in a fix set $N$

$$
Y=\left\{\left\langle n, \rho_{Y}(n), \sigma_{Y}(n), \lambda_{Y}(n) \mid n \in N\right\rangle\right\},
$$

where $\rho_{Y}(n), \quad \sigma_{Y}(n), \lambda_{Y}(n)$ are lying in $] 0^{-}, 1^{+}\left[\right.$and $0^{-} \leq \sup \rho_{Y}(n)+\sup \sigma_{Y}(n)+$ $\sup \lambda_{Y}(n) \leq 3^{+}$.

Definition 2 (Wang et al., 2004). An INS $\tilde{Y}$ in a fix set $N$ can be expressed:

$$
\tilde{Y}=\left\{\left\langle n, \tilde{\rho}_{\tilde{Y}}(n), \tilde{\sigma}_{\tilde{Y}}(n), \tilde{\lambda}_{\tilde{Y}}(n) \mid n \in N\right\rangle\right\},
$$

where $\tilde{\rho}_{\tilde{Y}}(n)=\left[\rho_{\tilde{Y}}^{L}(n), \rho_{\tilde{Y}}^{U}(n)\right] \subseteq[0,1], \tilde{\sigma}_{\tilde{Y}}(n)=\left[\sigma_{\tilde{Y}}^{L}(n), \sigma_{\tilde{Y}}^{U}(n)\right] \subseteq[0,1]$ and $\tilde{\lambda}_{\tilde{Y}}(n)=\left[\lambda_{\tilde{Y}}^{L}(n)\right.$, $\left.\lambda_{\tilde{Y}}^{U}(n)\right] \subseteq[0,1]$ represent truth membership, indeterminacy membership and falsity membership respectively, satisfying $0 \leq \rho_{\tilde{Y}}^{U}(n)+\sigma_{\tilde{Y}}^{U}(n)+\lambda_{\tilde{Y}}^{U}(n) \leq 3$.

Generally speaking, interval neutrosophic number $(\mathrm{INN}) \tilde{Y}=\left(\tilde{\rho}_{\tilde{Y}}, \tilde{\sigma}_{\tilde{Y}}, \tilde{\lambda}_{\tilde{Y}}\right)=\left(\left[\rho_{\tilde{Y}}^{L}, \rho_{\tilde{Y}}^{U}\right]\right.$, $\left.\left[\sigma_{\tilde{Y}}^{L}, \sigma_{\tilde{Y}}^{U}\right],\left[\lambda_{\tilde{Y}}^{L}, \lambda_{\tilde{Y}}^{U}\right]\right)$, the convenient form of INS, is a usual expression in calculating. Moreover, the score function and the accuracy function are created to describe relative precision.

Definition 3 (Tang, 2016). The score of INN $\tilde{Y}=\left(\left[\rho_{\tilde{Y}}^{L}, \rho_{\tilde{Y}}^{U}\right],\left[\sigma_{\tilde{Y}}^{L}, \sigma_{\tilde{Y}}^{U}\right],\left[\lambda \lambda_{\tilde{Y}}^{L}, \lambda \lambda_{\tilde{Y}}^{U}\right]\right)$ is

$$
S(\tilde{Y})=\frac{1}{6}\left[\left(2+\rho_{\tilde{Y}}^{L}-\sigma_{\tilde{Y}}^{L}-\lambda \lambda_{\tilde{Y}}^{L}\right)+\left(2+\rho_{\tilde{Y}}^{U}-\sigma_{\tilde{Y}}^{U}-\lambda \tilde{\tilde{Y}}_{\tilde{Y}}^{U}\right)\right], S(\tilde{Y}) \in[0,1] .
$$

Definition 4 (Tang, 2016). The accuracy function of INN $\tilde{Y}=\left(\left[\rho_{\tilde{Y}}^{L}, \rho_{\tilde{Y}}^{U}\right],\left[\sigma_{\tilde{Y}}^{L}, \sigma_{\tilde{Y}}^{U}\right],\left[\lambda_{\tilde{Y}}^{L}, \lambda \lambda_{\tilde{Y}}^{U}\right]\right)$ is 


$$
A(\tilde{Y})=\frac{1}{2}\left[\left(\rho_{\tilde{Y}}^{L}+\rho_{\tilde{Y}}^{U}\right)-\left(\lambda_{\tilde{Y}}^{L}+\lambda_{\tilde{Y}}^{U}\right)\right], A(\tilde{Y}) \in[-1,1] .
$$

Definition 5 (Tang, 2016). There are two INNs $\tilde{Y}=\left(\left[\rho_{\tilde{Y}}^{L}, \rho_{\tilde{Y}}^{U}\right],\left[\sigma_{\tilde{Y}}^{L}, \sigma_{\tilde{Y}}^{U}\right],\left[\lambda_{\tilde{Y}}^{L}, \lambda \lambda_{\tilde{Y}}^{U}\right]\right)$ and $\tilde{X}=\left(\left[\rho_{\tilde{X}}^{L}, \rho_{\tilde{X}}^{U}\right],\left[\sigma_{\tilde{X}}^{L}, \sigma_{\tilde{X}}^{U}\right],\left[\lambda \tilde{X}_{\tilde{X}}^{L}, \lambda \lambda_{\tilde{X}}^{U}\right]\right)$.

(1) If $S(\tilde{Y})>S(\tilde{X})$, then $\tilde{Y}>\tilde{X}$;

(2) If $S(\tilde{Y})=S(\tilde{X})$, then we need to compare the accuracy function of $\tilde{Y}$ and $\tilde{X}$;

(i) If $A(\tilde{Y})>A(\tilde{X})$, then $\tilde{Y}>\tilde{X}$;

(ii) If $A(\tilde{Y})=A(\tilde{X})$, then $\tilde{Y}=\tilde{X}$.

Definition 6 (Duong et al., 2020). Let $\tilde{Y}=\left(\left[\rho_{\tilde{Y}}^{L}, \rho_{\tilde{Y}}^{U}\right],\left[\sigma_{\tilde{Y}}^{L}, \sigma_{\tilde{Y}}^{U}\right],\left[\lambda \lambda_{\tilde{Y}}^{L}, \lambda \lambda_{\tilde{Y}}^{U}\right]\right)$ and $\tilde{X}=\left(\left[\rho_{\tilde{X}}^{L}, \rho_{\tilde{X}}^{U}\right]\right.$, $\left.\left[\sigma_{\tilde{X}}^{L}, \sigma_{\tilde{X}}^{U}\right],\left[\lambda \lambda_{\tilde{X}}^{L}, \lambda \tilde{X}_{\tilde{X}}^{U}\right]\right)$ be two INNs, and the following shows some basic operations of INNs.

(1) $\quad \tilde{Y}^{c}=\left(\left[\lambda_{\tilde{Y}}^{L}, \lambda \lambda_{\tilde{Y}}^{U}\right],\left[1-\sigma_{\tilde{Y}}^{U}, 1-\sigma_{\tilde{Y}}^{L}\right],\left[\rho_{\tilde{Y}}^{L}, \rho_{\tilde{Y}}^{U}\right]\right)$;

(2) $\beta \tilde{Y}=\left(\left[1-\left(1-\rho_{\tilde{Y}}^{L}\right)^{\beta}, 1-\left(1-\rho_{\tilde{Y}}^{U}\right)^{\beta}\right],\left[\left(\sigma_{\tilde{Y}}^{L}\right)^{\beta},\left(\sigma_{\tilde{Y}}^{U}\right)^{\beta}\right],\left[\left(\lambda_{\tilde{Y}}^{L}\right)^{\beta},\left(\lambda_{\tilde{Y}}^{U}\right)^{\beta}\right]\right), \beta>0$;

(3) $(\tilde{Y})^{\beta}=\left(\left[\left(\rho_{\tilde{Y}}^{L}\right)^{\beta},\left(\rho_{\tilde{Y}}^{U}\right)^{\beta}\right],\left[\left(\sigma_{\tilde{Y}}^{L}\right)^{\beta},\left(\sigma_{\tilde{Y}}^{U}\right)^{\beta}\right],\left[1-\left(1-\lambda_{\tilde{Y}}^{L}\right)^{\beta}, 1-\left(1-\lambda_{\tilde{Y}}^{U}\right)^{\beta}\right]\right), \beta>0$;

(4) $\tilde{Y} \oplus \tilde{X}=\left(\left[\rho_{\tilde{Y}}^{L}+\rho_{\tilde{X}}^{L}-\rho_{\tilde{Y}}^{L} \rho_{\tilde{X}}^{L}, \rho_{\tilde{Y}}^{U}+\rho_{\tilde{X}}^{U}-\rho_{\tilde{Y}}^{U} \rho_{\tilde{X}}^{U}\right],\left[\sigma_{\tilde{Y}}^{L} \sigma_{\tilde{X}}^{L}, \sigma_{\tilde{Y}}^{U} \sigma_{\tilde{X}}^{U}\right],\left[\lambda \lambda_{\tilde{Y}}^{L} \lambda \lambda_{\tilde{X}}^{L}, \lambda \lambda_{\tilde{Y}}^{U} \lambda \lambda_{\tilde{X}}^{U}\right]\right)$;

(5) $\quad \tilde{Y} \otimes \tilde{X}=\left(\begin{array}{l}\left.\left[\rho_{\tilde{Y}}^{L} \rho_{\tilde{X}}^{L}, \rho_{\tilde{Y}}^{U} \rho_{\tilde{X}}^{U}\right],\left[\sigma_{\tilde{Y}}^{L}+\sigma_{\tilde{X}}^{L}-\sigma_{\tilde{Y}}^{L} \sigma_{\tilde{X}}^{L}, \sigma_{\tilde{Y}}^{U}+\sigma_{\tilde{X}}^{U}-\sigma_{\tilde{Y}}^{U} \sigma_{\tilde{X}}^{U}\right],\right) \\ {\left[\lambda_{\tilde{Y}}^{L}+\lambda \lambda_{\tilde{X}}^{L}-\lambda \lambda_{\tilde{Y}}^{L} \lambda_{\tilde{X}}^{L}, \lambda \lambda_{\tilde{Y}}^{U}+\lambda \tilde{X}_{\tilde{X}}^{U}-\lambda \lambda_{\tilde{Y}}^{U} \lambda_{\tilde{X}}^{U}\right]}\end{array}\right)$.

Definition 7 (Tang, 2016). Let $\tilde{Y}=\left(\left[\rho_{\tilde{Y}}^{L}, \rho_{\tilde{Y}}^{U}\right],\left[\sigma_{\tilde{Y}}^{L}, \sigma_{\tilde{Y}}^{U}\right],\left[\lambda \lambda_{\tilde{Y}}^{L}, \lambda \lambda_{\tilde{Y}}^{U}\right]\right)$ and $\tilde{X}=\left(\left[\rho_{\tilde{X}}^{L}, \rho_{\tilde{X}}^{U}\right]\right.$, $\left.\left[\lambda \lambda_{\tilde{X}}^{L}, \lambda \lambda_{\tilde{X}}^{U}\right]\right)$ be two INNs, the normalized Hamming distance between these two INNs can be acquired by the following Eq. (5):

$$
d(\tilde{Y}, \tilde{X})=\frac{\left(\left|\rho_{\tilde{Y}}^{L}-\rho_{\tilde{X}}^{L}\right|+\left|\rho_{\tilde{Y}}^{U}-\rho_{\tilde{X}}^{U}\right|\right)+\left(\left|\sigma_{\tilde{Y}}^{L}-\sigma_{\tilde{X}}^{L}\right|+\left|\sigma_{\tilde{Y}}^{U}-\sigma_{\tilde{X}}^{U}\right|\right)+\left(\left|\lambda_{\tilde{Y}}^{L}-\lambda \lambda_{\tilde{X}}^{L}\right|+\left|\lambda_{\tilde{Y}}^{U}-\lambda \tilde{X}_{\tilde{X}}^{U}\right|\right)}{6} .
$$

Definition 8 (Garg \& Nancy, 2020). If there is a collection of INNs $\tilde{Y}_{l}=\left(\left[\rho_{\tilde{Y}_{l}}^{L}, \rho_{\tilde{Y}_{l}}^{U}\right],\left[\sigma_{\tilde{Y}_{l}}^{L}, \sigma_{\tilde{Y}_{l}}^{U}\right]\right.$, $\left.\left[\lambda{\tilde{\tilde{Y}_{l}}}_{l}^{L}, \lambda_{\tilde{Y}_{l}}^{U}\right]\right)(l=1,2, \ldots, f)$ and the weighting vector of $\tilde{Y}_{l}(l=1,2, \ldots, f)$ is $\alpha=\left(\alpha_{1}, \alpha_{2}, \cdots, \alpha_{f}\right)^{T}$ where $\alpha_{l} \geq 0$ and $\sum_{l=1}^{f} \alpha_{l}=1$, then the interval neutrosophic weighted averaging (INWA) aggregation operator is

$$
\begin{aligned}
& \operatorname{INWA}_{\alpha}\left(\tilde{Y}_{1}, \tilde{Y}_{2}, \cdots, \tilde{Y}_{f}\right)=\underset{l=1}{f}\left(\alpha_{l} \tilde{Y}_{l}\right) \\
& =\left(\begin{array}{l}
{\left[1-\prod_{l=1}^{f}\left(1-\rho_{\tilde{Y}_{l}}^{L}\right)^{\alpha_{l}}, 1-\prod_{l=1}^{f}\left(1-\rho_{\tilde{Y}_{l}}^{U}\right)^{\alpha_{l}}\right],} \\
{\left[\prod_{l=1}^{f}\left(\sigma_{\tilde{Y}_{l}}^{L}\right)^{\alpha_{l}}, \prod_{l=1}^{f}\left(\sigma_{\tilde{Y}_{l}}^{U}\right)^{\alpha_{l}}\right],\left[\prod_{l=1}^{f}\left(\lambda_{\tilde{Y}_{l}}^{L}\right)^{\alpha_{l}}, \prod_{l=1}^{f}\left(\lambda_{\tilde{Y}_{l}}^{U}\right)^{\alpha_{l}},\right]}
\end{array}\right) .
\end{aligned}
$$


Definition 9 (Garg \& Nancy, 2020). If there is a collection of INNs $\tilde{Y}_{l}=\left(\left[\rho_{\tilde{Y}_{l}}^{L}, \rho_{\tilde{Y}_{l}}^{U}\right]\right.$, $\left.\left[\sigma_{\tilde{Y}_{l}}^{L}, \sigma_{\tilde{Y}_{l}}^{U}\right],\left[\lambda_{\tilde{Y}_{l}}^{L}, \lambda{\tilde{Y_{l}}}_{l}^{U}\right]\right)(l=1,2, \ldots, f)$ and the weighting vector of $\tilde{Y}_{l}(l=1,2, \ldots, f)$ is $\alpha=\left(\alpha_{1}, \alpha_{2}, \cdots, \alpha_{f}\right)^{T}$ where $\alpha_{l} \geq 0$ and $\sum_{l=1}^{f} \alpha_{l}=1$, then the interval neutrosophic weighted geometric (INWG) aggregation operator is

$$
\begin{aligned}
& \operatorname{INWG}_{\alpha}\left(\tilde{Y}_{1}, \tilde{Y}_{2}, \cdots, \tilde{Y}_{f}\right)=\underset{l=1}{\otimes}\left(\alpha_{l} \tilde{Y}_{l}\right) \\
& =\left(\begin{array}{l}
{\left[\prod_{l=1}^{f}\left(\rho_{\tilde{Y}_{l}}^{L}\right)^{\alpha_{l}}, \prod_{l=1}^{f}\left(\rho_{\tilde{Y}_{l}}^{U}\right)^{\alpha_{l}}\right],\left[1-\prod_{l=1}^{f}\left(1-\sigma_{\tilde{Y}_{l}}^{L}\right)^{\alpha_{l}}, 1-\prod_{l=1}^{f}\left(1-\sigma_{\tilde{Y}_{l}}^{U}\right)^{\alpha_{l}}\right],} \\
{\left[1-\prod_{l=1}^{f}\left(1-\lambda \tilde{Y}_{l}^{L}\right)^{\alpha_{l}}, 1-\prod_{l=1}^{f}\left(1-\lambda \tilde{Y}_{l}^{U}\right)^{\alpha_{l}}\right]}
\end{array}\right)
\end{aligned}
$$

\section{Interval neutrosophic TODIM based on cumulative prospect theory}

In this topic, we introduce a novel MAGDM method named the CPT-IN-TODIM method. Suppose there are three collections $T=\left\{T_{1}, T_{2}, \cdots, T_{h}\right\}, B=\left\{B_{1}, B_{2}, \cdots, B_{p}\right\}$ and $D=\left\{D_{1}, D_{2}, \cdots, D_{f}\right\}$, which stand for alternatives, attributes and decision makers respectively. At the same time, the weighting vector of DMs is $\alpha=\left(\alpha_{1}, \alpha_{2}, \cdots, \alpha_{f}\right)^{T}$ where $\alpha_{l} \geq 0$ and $\sum_{l=1}^{f} \alpha_{l}=1$. The weighting vector of attributes is unknown, but it is expressed as $\mathfrak{l}=\left(\mathrm{l}_{1}, \mathrm{l}_{2}, \ldots, \mathrm{l}_{p}\right)^{T}\left(\mathrm{l}_{o} \geq 0\right.$ and $\left.\sum_{o=1}^{p} \mathrm{l}_{o}=1\right)$.

Step 1. Build the interval neutrosophic decision matrix $\tilde{Z}^{(l)}=\left(\tilde{z}_{k o}^{(l)}\right)_{h \times p}=\left(\left\langle\tilde{\rho}_{k o}^{(l)}, \tilde{\sigma}_{k o}^{(l)}, \tilde{\lambda}_{k o}^{(l)}\right\rangle\right)_{h \times p}$ of the decision maker $D_{l}$, where indicate truth-membership degree $\tilde{\rho}_{k o}^{(l)}=\left[\rho_{k o}^{L}(l), \rho_{k o}^{U}(l)\right]$, indeterminacy-membership degree $\tilde{\sigma}_{k o}^{(l)}=\left[\sigma_{k o}^{L}(l), \sigma_{k o}^{U}(l)\right]$ and falsity-membership degree $\tilde{\lambda}_{k o}^{(l)}=\left[\lambda_{k o}^{L}(l), \lambda_{k o}^{U}(l)\right]$ and all of them satisfy $\tilde{\rho}_{k o}^{(l)}, \tilde{\sigma}_{k o}^{(l)}, \tilde{\lambda}_{k o}^{(l)} \subseteq[0,1]$ and $0 \leq \tilde{\rho}_{k o}^{(l)}+\tilde{\sigma}_{k o}^{(l)}+\tilde{\lambda}_{k o}^{(l)} \leq 3$ $(k=1,2, \cdots, h, o=1,2, \cdots, p, l=1,2, \cdots, f)$.

Step 2. Translate negative attributes by using Eq. (8) to ensure consistency of measurement scale and finally make up the standardized interval neutrosophic decision matrix $\tilde{G}^{(l)}=\left(\tilde{g}_{k o}^{(l)}\right)_{h \times p}(k=1,2, \cdots, h, o=1,2, \cdots, p, l=1,2, \cdots, f)$. 


$$
\begin{aligned}
& \tilde{g}_{k o}^{(l)}=\left\langle\tilde{v}_{k o}^{(l)}, \tilde{\varphi}_{k o}^{(l)}, \tilde{\varepsilon}_{k o}^{(l)}\right\rangle=\left\langle\left[v_{k o}^{L}(l), v_{k o}^{U}(l)\right],\left[\varphi_{k o}^{L}(l), \varphi_{k o}^{U}(l)\right],\left[\varepsilon_{k o}^{L}(l), \varepsilon_{k o}^{U}(l)\right]\right\rangle \\
& \tilde{z}_{k o}^{(l)}=\left\langle\tilde{\rho}_{k o}^{(l)}, \tilde{\sigma}_{k o}^{(l)}, \tilde{\lambda}_{k o}^{(l)}\right\rangle, T_{k} \text { is a positive attribute } \\
& =\left\langle\begin{array}{l}
\left.\left[\rho_{k o}^{L}(l), \rho_{k o}^{U}(l)\right],\left[\sigma_{k o}^{L}(l), \sigma_{k o}^{U}(l)\right],\right\rangle \\
{\left[\lambda_{k o}^{L}(l), \lambda_{k o}^{U}(l)\right]}
\end{array}\right], T_{k} \text { is a negative attribute } \\
& =\left\langle\begin{array}{l}
\left.\tilde{z}_{k o}^{(l)}\right)^{c}=\left\langle\tilde{\lambda}_{k o}^{(l)}, 1-\tilde{\sigma}_{k o}^{(l)}, \tilde{\rho}_{k o}^{(l)}\right\rangle \\
{\left[\lambda_{k o}^{L}(l), \lambda_{k o}^{U}(l)\right],\left[1-\sigma_{k o}^{U}(l), 1-\sigma_{k o}^{L}(l)\right],} \\
{\left[\rho_{k o}^{L}(l), \rho_{k o}^{U}(l)\right]}
\end{array}\right.
\end{aligned}
$$

Step 3. The INWA operator is used to integrate all decision matrices from different decision makers into one group decision matrix $\tilde{Q}=\left(\tilde{q}_{k o}\right)_{h \times p}$, shown as the Eq. (9):

$$
\begin{aligned}
\tilde{q}_{k o}= & \left\langle\tilde{v}_{k o}, \tilde{\varphi}_{k o}, \tilde{\varepsilon}_{k o}\right\rangle=\left\langle\left[v_{k o}^{L}, v_{k o}^{U}\right],\left[\varphi_{k o}^{L}, \varphi_{k o}^{U}\right],\left[\varepsilon_{k o}^{L}, \varepsilon_{k o}^{U}\right]\right\rangle \\
= & \operatorname{INWA}_{\alpha}\left(\tilde{g}_{k o}^{(1)}, \tilde{g}_{k o}^{(2)}, \ldots, \tilde{g}_{k o}^{(f)}\right)=\oplus_{l=1}^{f}\left(\alpha_{l} \tilde{g}_{k o}^{(l)}\right) \\
& \left\langle\left[\begin{array}{l}
{\left[1-\prod_{l=1}^{f}\left(1-v_{k o}^{L}(l)\right)^{\alpha_{l}}, 1-\prod_{l=1}^{f}\left(1-v_{k o}^{U}(l)\right)^{\alpha_{l}}\right],} \\
\left.\left[\prod_{l=1}^{f}\left(\varphi_{k o}^{L}(l)\right)^{\alpha_{l}}, \prod_{l=1}^{f}\left(\varphi_{k o}^{U}(l)\right)^{\alpha_{l}}\right],\left[\prod_{l=1}^{f}\left(\varepsilon_{k o}^{L}(l)\right)^{\alpha_{l}}, \prod_{l=1}^{f}\left(\varepsilon_{k o}^{U}(l)\right)^{\alpha_{l}},\right]\right\rangle
\end{array} .\right.\right.
\end{aligned}
$$

Step 4. The entropy weight method, just as equation (10) and (11), helps to achieve the initial weighting vector of attributes $\mathrm{l}=\left(\mathrm{l}_{1}, \mathrm{l}_{2}, \ldots, \mathrm{l}_{p}\right)^{T}\left(\mathrm{t}_{o} \geq 0\right.$ and $\left.\sum_{o=1}^{p} \mathrm{t}_{o}=1\right)$ in line with the known information that the decision matrix $\tilde{Q}=\left(\tilde{q}_{k o}\right)_{h \times p}$ gives us.

$$
\begin{gathered}
E_{o}=-\frac{1}{\ln h} \sum_{k=1}^{h} S\left(\tilde{q}_{k o}\right) \ln \left(S\left(\tilde{q}_{k o}\right)\right) ; \\
\mathrm{l}_{o}=\frac{1-E_{o}}{\sum_{o=1}^{p}\left(1-E_{o}\right)}, o=1,2, \cdots, p .
\end{gathered}
$$

Step 5. The weighting function (12) and Eq. (13) are taking advantage of disposing the initial weighting vector of attributes $\mathrm{l}=\left(\mathrm{l}_{1}, \mathrm{l}_{2}, \ldots, \mathrm{l}_{p}\right)^{T}$ to obtain the modified weights $\partial_{\text {kio }}^{*}\left(\mathrm{l}_{o}\right)$ $(k, i=1,2, \cdots, h ; o=1,2, \cdots, p)$.

$$
\partial_{k i o}\left(\mathrm{l}_{o}\right)= \begin{cases}\left(\mathrm{l}_{o}\right)^{a} /\left(\left(\mathrm{t}_{o}\right)^{a}+\left(1-\mathrm{s}_{o}\right)^{a}\right)^{\frac{1}{a}}, & \tilde{q}_{k o} \geq \tilde{q}_{i o} \\ \left(\mathrm{l}_{o}\right)^{b} /\left(\left(\mathrm{t}_{o}\right)^{b}+\left(1-\mathrm{l}_{o}\right)^{b}\right)^{\frac{1}{b}}, & \tilde{q}_{k o}<\tilde{q}_{i o}\end{cases}
$$




$$
\partial_{k i o}^{*}\left(\mathrm{l}_{o}\right)=\frac{\partial_{k i o}\left(\mathrm{l}_{o}\right)}{\max \left\{\partial_{k i t}\left(\mathrm{l}_{t}\right) \mid t \in p\right\}}, \forall k, i \in h ; 0=1,2, \cdots, p ;
$$

Step 6. Compare the differences under each attribute between any two alternatives based on Eqs (14)-(15), just as $\delta_{o}\left(T_{k}, T_{i}\right)$ means the relative predominance of alternative $T_{k}$ as compared to alternative $T_{i}$ under the attribute $B_{o}$. And then utilize Eq. (16) to put the holistic preponderance of alternative $T_{k}$ compared with alternative $T_{i}$ together.

$$
\begin{aligned}
& d_{o}\left(T_{k}, T_{i}\right)=\frac{1}{6}\left(\begin{array}{l}
\left(\left|v_{k o}^{L}-v_{i o}^{L}\right|+\left|v_{k o}^{U}-v_{i o}^{U}\right|\right)+ \\
\left(\left|\varphi_{k o}^{L}-\varphi_{i o}^{L}\right|+\left|\varphi_{k o}^{U}-\varphi_{i o}^{U}\right|\right)+ \\
\left(\left|\varepsilon_{k o}^{L}-\varepsilon_{i o}^{L}\right|+\left|\varepsilon_{k o}^{U}-\varepsilon_{i o}^{U}\right|\right)
\end{array}\right), k, i=1,2, \cdots, h ; \\
& \delta_{o}\left(T_{k}, T_{i}\right)= \begin{cases}\frac{\partial_{k i o}^{*}\left(\mathrm{~s}_{o}\right) \cdot\left(d_{o}\left(T_{k}, T_{i}\right)\right)^{\eta}}{\sum_{o=1}^{p} \partial_{k i o}^{*}\left(\mathrm{~s}_{o}\right)}, & \text { if } \tilde{q}_{k o}>\tilde{q}_{k o} \\
0 & \text { if } \tilde{q}_{k o}=\tilde{q}_{k o} ; \\
-\theta \cdot \frac{\left(\sum_{o=1}^{p} \partial_{k i o}^{*}\left(\mathrm{l}_{o}\right)\right) \cdot\left(d_{o}\left(T_{k}, T_{i}\right)\right)^{\mu}}{\partial_{k i o}^{*}\left(\mathrm{~s}_{o}\right)}, & \text { if } \tilde{q}_{k o}<\tilde{q}_{k o}\end{cases} \\
& \wp\left(T_{k}, T_{i}\right)=\sum_{o=1}^{p} \delta_{o}\left(T_{k}, T_{i}\right), k, i=1,2, \ldots, p,
\end{aligned}
$$

where $\theta, \eta$ and $\mu$ are the parameters. And we can show the relative predominance $\delta_{o}\left(T_{k}, T_{i}\right)$ and the overall predominance $\wp\left(T_{k}, T_{i}\right)$ in matrix form, just as the relative predominance matrix $\delta_{o}=\left(\delta_{o}\left(T_{k}, T_{i}\right)\right)_{h \times h}$ and the overall predominance matrix $\wp=\left(\wp\left(T_{k}, T_{i}\right)\right)_{h \times h}$ $(k, i=1,2, \cdots, h)$.

$$
\begin{aligned}
& \begin{array}{ccccc}
T_{1} & T_{2} & \cdots & T_{h}
\end{array} \\
& \delta_{o}=\left(\delta_{o}\left(T_{k}, T_{i}\right)\right)_{h \times h}=\begin{array}{c}
T_{1} \\
T_{2} \\
\vdots \\
T_{h}
\end{array}\left(\begin{array}{cccc}
0 & \delta_{o}\left(T_{1}, T_{2}\right) & \cdots & \delta_{o}\left(T_{1}, T_{h}\right) \\
\delta_{o}\left(T_{2}, T_{1}\right) & 0 & \cdots & \delta_{o}\left(T_{2}, T_{h}\right) \\
\vdots & \vdots & \ddots & \vdots \\
\delta_{o}\left(T_{h}, T_{1}\right) & \delta_{o}\left(T_{h}, T_{2}\right) & \cdots & 0
\end{array}\right) ; \\
& o=1,2, \cdots, p
\end{aligned}
$$

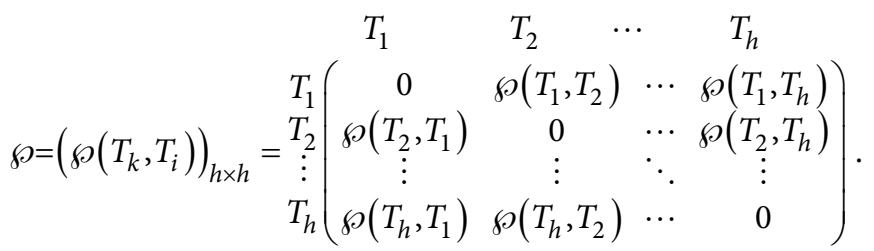

$$
\begin{aligned}
& o=1,2, \cdots, p
\end{aligned}
$$

Step 7. In line with Eq. (19), standardize the overall predominance $\wp\left(T_{k}, T_{i}\right)$ to acquire the standard overall predominance $\mathfrak{I}\left(T_{k}\right)(k=1,2, \ldots, h)$ for the sake of comparison. The greater the standard overall predominance is, the better the alternative is. 


$$
\mathfrak{I}\left(T_{k}\right)=\frac{\sum_{i=1}^{h} \wp\left(T_{k}, T_{i}\right)-\min _{k}\left\{\sum_{i=1}^{h} \wp\left(T_{k}, T_{i}\right)\right\}}{\max _{k}\left\{\sum_{i=1}^{h} \wp\left(T_{k}, T_{i}\right)\right\}-\min _{k}\left\{\sum_{i=1}^{h} \wp\left(T_{k}, T_{i}\right)\right\}} \quad k=1,2, \ldots, h .
$$

\section{Numerical instance}

In this part, we tend to apply this new CPT-IN-TODIM method to give companies a hand assessing third-party cold chain logistics service providers. Now, there are four cold chain logistics service providers $T_{k}(k=1,2,3,4)$ awaiting evaluation. Three experts $D_{l}(l=1,2,3)$ are invited to analyze five aspects of these providers: (1) $B_{1}$ is the degree of wastage reduction of fresh agricultural products; (2) $B_{2}$ is the degree of advanced service concept; (3) $B_{3}$ is the technical level of cold chain; (4) $B_{4}$ is the intelligence degree of cold chain information system; (5) $B_{5}$ is the distance from logistics point to producing area of fresh agricultural products. Additionally, the weighting vector of experts is $\alpha=\left(\alpha_{1}, \alpha_{2}, \alpha_{3}\right)^{T}=(0.4,0.25,0.35)^{T}$. And each expert's assessment of these systems is depicted in the following Table 1 to Table 3.

Table 1. Decision matrix $\tilde{Z}^{(1)}$ given by the expert $D_{1}$

\begin{tabular}{|c|c|c|c|c|c|}
\hline & $B_{1}$ & $B_{2}$ & $B_{3}$ & $B_{4}$ & $B_{5}$ \\
\hline$T_{1}$ & $\left\langle\begin{array}{l}{[0.45,0.55],} \\
{[0.25,0.30]} \\
{[0.35,0.45]}\end{array}\right\rangle$ & $\left\langle\begin{array}{l}{[0.70,0.80],} \\
{[0.15,0.20],} \\
{[0.25,0.35]}\end{array}\right\rangle$ & $\left\langle\begin{array}{l}{[0.55,0.65],} \\
{[0.25,0.30],} \\
{[0.35,0.50]}\end{array}\right\rangle$ & $\begin{array}{l}{\left[\begin{array}{l}{[0.65,0.70]} \\
{[0.30,0.40]} \\
{[0.40,0.50]}\end{array}\right\rangle}\end{array}$ & $\begin{array}{l}\langle[0.30,0.40], \\
{[0.50,0.60]} \\
{[0.45,0.50]}\end{array}$ \\
\hline$T_{2}$ & $\left\langle\begin{array}{l}{[0.70,0.85]} \\
{[0.10,0.15]} \\
{[0.15,0.25]}\end{array}\right\rangle$ & $\left\langle\begin{array}{l}{[0.65,0.70],} \\
{[0.25,0.30]} \\
{[0.30,0.40]}\end{array}\right\rangle$ & $\left\langle\begin{array}{l}{[0.75,0.85],} \\
{[0.15,0.20]} \\
{[0.20,0.25]}\end{array}\right\rangle$ & $\left.\begin{array}{l}{[0.70,0.80],} \\
{[0.25,0.30],} \\
{[0.30,0.40]}\end{array}\right\rangle$ & $\left.\begin{array}{l}{[0.20,0.25],} \\
{[0.65,0.70],} \\
{[0.65,0.70]}\end{array}\right\rangle$ \\
\hline$T_{3}$ & 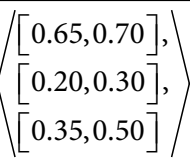 & $\begin{array}{l}{[0.60,0.75],} \\
{[0.20,0.30],} \\
{[0.25,0.35]}\end{array}$ & $\left\langle\begin{array}{l}{[0.70,0.80],} \\
{[0.20,0.25],} \\
{[0.30,0.35]}\end{array}\right\rangle$ & $\begin{array}{l}{[0.65,0.75],} \\
{[0.30,0.35],} \\
{[0.35,0.45]}\end{array}$ & $\begin{array}{l}{[0.15,0.25],} \\
{[0.70,0.80],} \\
{[0.75,0.80]}\end{array}$ \\
\hline$T_{4}$ & $\left.\begin{array}{l}{[0.50,0.55],} \\
{[0.25,0.35]} \\
{[0.40,0.45]}\end{array}\right\rangle$ & $\begin{array}{l}{[0.65,0.75],} \\
{[0.25,0.35]} \\
{[0.20,0.25]}\end{array}$ & $\left\langle\begin{array}{l}{[0.60,0.70],} \\
{[0.25,0.35],} \\
{[0.35,0.40]}\end{array}\right\rangle$ & $\begin{array}{l}{[0.70,0.75],} \\
{[0.25,0.35],} \\
{[0.35,0.40]}\end{array}$ & $\left.\begin{array}{l}\langle[0.35,0.45], \\
{[0.55,0.65]} \\
{[0.50,0.60]}\end{array}\right\rangle$ \\
\hline
\end{tabular}


Table 2. Decision matrix $\tilde{Z}^{(2)}$ given by the expert $D_{2}$

\begin{tabular}{|c|c|c|c|c|c|}
\hline & $B_{1}$ & $B_{2}$ & $B_{3}$ & $B_{4}$ & $B_{5}$ \\
\hline$T_{1}$ & $\left\langle\begin{array}{l}{[0.45,0.55],} \\
{[0.25,0.30],} \\
{[0.35,0.45]}\end{array}\right\rangle$ & $\left\langle\begin{array}{l}{[0.65,0.75],} \\
{[0.20,0.25],} \\
{[0.25,0.35]}\end{array}\right\rangle$ & $\left\langle\begin{array}{l}{[0.65,0.70],} \\
{[0.30,0.35],} \\
{[0.35,0.45]}\end{array}\right\rangle$ & $\left\langle\begin{array}{l}{[0.60,0.70],} \\
{[0.25,0.40],} \\
{[0.40,0.45]}\end{array}\right\rangle$ & $\begin{array}{l}\langle[0.30,0.35], \\
{[0.60,0.65]} \\
{[0.50,0.65]}\end{array}$ \\
\hline$T_{2}$ & $\left\langle\begin{array}{l}{[0.70,0.85],} \\
{[0.10,0.15],} \\
{[0.15,0.25]}\end{array}\right\rangle$ & $\left\langle\begin{array}{l}{[0.70,0.75],} \\
{[0.20,0.25],} \\
{[0.25,0.35]}\end{array}\right\rangle$ & $\left\langle\begin{array}{l}{[0.70,0.80],} \\
{[0.20,0.30],} \\
{[0.30,0.35]}\end{array}\right\rangle$ & $\left\langle\begin{array}{l}{[0.75,0.85],} \\
{[0.20,0.30],} \\
{[0.30,0.35]}\end{array}\right\rangle$ & $\begin{array}{l}{\left[\begin{array}{l}{[0.15,0.20]} \\
{[0.75,0.80]} \\
{[0.75,0.85]}\end{array}\right)}\end{array}$ \\
\hline$T_{3}$ & $\left(\begin{array}{l}{[0.65,0.70],} \\
{[0.20,0.30]} \\
{[0.35,0.50]}\end{array}\right)$ & $\left\langle\begin{array}{l}{[0.65,0.70],} \\
{[0.20,0.30]} \\
{[0.30,0.35]}\end{array}\right\rangle$ & $\left\langle\begin{array}{c}{[0.70,0.80],} \\
{[0.15,0.25],} \\
{[0.25,0.30]}\end{array}\right\rangle$ & $\left\langle\begin{array}{l}{[0.75,0.80],} \\
{[0.25,0.30],} \\
{[0.35,0.40]}\end{array}\right\rangle$ & 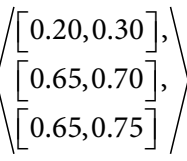 \\
\hline$T_{4}$ & $\left\langle\begin{array}{l}{[0.50,0.55],} \\
{[0.25,0.35],} \\
{[0.40,0.45]}\end{array}\right\rangle$ & $\left\langle\begin{array}{l}{[0.60,0.65],} \\
{[0.25,0.30],} \\
{[0.30,0.40]}\end{array}\right\rangle$ & $\left\langle\begin{array}{l}{[0.65,0.75],} \\
{[0.20,0.25],} \\
{[0.30,0.40]}\end{array}\right\rangle$ & $\left\langle\begin{array}{l}{[0.65,0.75],} \\
{[0.30,0.35]} \\
{[0.40,0.50]}\end{array}\right\rangle$ & $\begin{array}{l}{\left[\begin{array}{l}{[0.30,0.35]} \\
{[0.65,0.75]} \\
{[0.55,0.65]}\end{array}\right)} \\
\end{array}$ \\
\hline
\end{tabular}

Table 3. Decision matrix $\tilde{Z}^{(3)}$ given by the expert $D_{3}$

\begin{tabular}{|c|c|c|c|c|c|}
\hline & $B_{1}$ & $B_{2}$ & $B_{3}$ & $B_{4}$ & $B_{5}$ \\
\hline$T_{1}$ & $\left\langle\begin{array}{l}{[0.45,0.55],} \\
{[0.25,0.30]} \\
{[0.35,0.45]}\end{array}\right\rangle$ & $\left\langle\begin{array}{l}{[0.75,0.80],} \\
{[0.15,0.20]} \\
{[0.20,0.25]}\end{array}\right\rangle$ & $\left\langle\begin{array}{l}{[0.65,0.75],} \\
{[0.30,0.40]} \\
{[0.40,0.45]}\end{array}\right\rangle$ & $\left\langle\begin{array}{l}{[0.65,0.75],} \\
{[0.30,0.35],} \\
{[0.35,0.40]}\end{array}\right\rangle$ & $\left\langle\begin{array}{l}{[0.25,0.35],} \\
{[0.65,0.70]} \\
{[0.60,0.65]}\end{array}\right\rangle$ \\
\hline$T_{2}$ & $\left\langle\begin{array}{l}{[0.70,0.85],} \\
{[0.10,0.15]} \\
{[0.15,0.25]}\end{array}\right\rangle$ & $\left\langle\begin{array}{l}{[0.60,0.70],} \\
{[0.30,0.35],} \\
{[0.35,0.45]}\end{array}\right\rangle$ & $\left\langle\begin{array}{l}{[0.70,0.80],} \\
{[0.25,0.30],} \\
{[0.30,0.40]}\end{array}\right\rangle$ & $\left\langle\begin{array}{l}{[0.70,0.85],} \\
{[0.20,0.30],} \\
{[0.30,0.35]}\end{array}\right\rangle$ & $\left\langle\begin{array}{l}{[0.20,0.30],} \\
{[0.70,0.75]} \\
{[0.75,0.80]}\end{array}\right\rangle$ \\
\hline$T_{3}$ & $\left(\begin{array}{l}{[0.65,0.70],} \\
{[0.20,0.30]} \\
{[0.35,0.50]}\end{array}\right)$ & $\left\langle\begin{array}{l}{[0.65,0.75],} \\
{[0.25,0.30]} \\
{[0.30,0.35]}\end{array}\right.$ & $\left\langle\begin{array}{l}{[0.75,0.85],} \\
{[0.20,0.25],} \\
{[0.25,0.35]}\end{array}\right\rangle$ & $\left\langle\begin{array}{l}{[0.75,0.80],} \\
{[0.20,0.25]} \\
{[0.30,0.40]}\end{array}\right\rangle$ & $\left\langle\begin{array}{l}{[0.10,0.20],} \\
{[0.80,0.90]} \\
{[0.80,0.85]}\end{array}\right\rangle$ \\
\hline$T_{4}$ & $\left\langle\begin{array}{l}{[0.50,0.55],} \\
{[0.25,0.35],} \\
{[0.40,0.45]}\end{array}\right\rangle$ & $\left\langle\begin{array}{l}{[0.70,0.75],} \\
{[0.20,0.30],} \\
{[0.25,0.35]}\end{array}\right\rangle$ & $\left\langle\begin{array}{l}{[0.60,0.65],} \\
{[0.35,0.45],} \\
{[0.45,0.50]}\end{array}\right\rangle$ & $\left\langle\begin{array}{l}{[0.60,0.70],} \\
{[0.35,0.40],} \\
{[0.40,0.50]}\end{array}\right\rangle$ & $\left.\begin{array}{l}{[0.25,0.35],} \\
{[0.55,0.60],} \\
{[0.55,0.65]}\end{array}\right\rangle$ \\
\hline
\end{tabular}

Step 1. Build the interval neutrosophic decision matrix $\tilde{Z}^{(l)}=\left(\tilde{z}_{k o}^{(l)}\right)_{4 \times 5}=\left(\left\langle\tilde{\rho}_{k o}^{(l)}, \tilde{\sigma}_{k o}^{(l)}, \tilde{\lambda}_{k o}^{(l)}\right\rangle\right)_{4 \times 5}$
$(l=1,2,3)$ just like the shown in Table 1 to Table 3.

Step 2. Translate negative attributes by using Eq. (8) to ensure consistency of measurement scale and finally make up the standardized interval neutrosophic decision matrix $\tilde{G}^{(l)}=\left(\tilde{g}_{k o}^{(l)}\right)_{4 \times 5}(l=1,2,3)$. Please see Tables $4-6$. 
Table 4. Standardized decision matrix $\tilde{G}^{(1)}$ given by the expert $D_{1}$

\begin{tabular}{|c|c|c|c|c|c|}
\hline & $B_{1}$ & $B_{2}$ & $B_{3}$ & $B_{4}$ & $B_{5}$ \\
\hline$T_{1}$ & $\begin{array}{l}{[0.45,0.55],} \\
{[0.25,0.30],} \\
{[0.35,0.45]}\end{array}$ & $\left\langle\begin{array}{l}{[0.70,0.80],} \\
{[0.15,0.20]} \\
{[0.25,0.35]}\end{array}\right\rangle$ & $\left\langle\begin{array}{l}{[0.55,0.65]} \\
{[0.25,0.30]} \\
{[0.35,0.50]}\end{array}\right\rangle$ & $\left\langle\begin{array}{l}{[0.65,0.70]} \\
{[0.30,0.40]} \\
{[0.40,0.50]}\end{array}\right\rangle$ & $\left.\begin{array}{l}([0.45,0.50], \\
{[0.40,0.50]} \\
{[0.30,0.40]}\end{array}\right\rangle$ \\
\hline$T_{2}$ & $\begin{array}{l}{[0.70,0.85],} \\
{[0.10,0.15]} \\
{[0.15,0.25]}\end{array}$ & $\left\langle\begin{array}{l}{[0.65,0.70]} \\
{[0.25,0.30]} \\
{[0.30,0.40]}\end{array}\right\rangle$ & $\left\langle\begin{array}{l}{[0.75,0.85],} \\
{[0.15,0.20]} \\
{[0.20,0.25]}\end{array}\right\rangle$ & $\left\langle\begin{array}{l}{[0.70,0.80]} \\
{[0.25,0.30]} \\
{[0.30,0.40]}\end{array}\right\rangle$ & $\left.\begin{array}{l}0.65,0.70], \\
0.30,0.35] \\
0.20,0.25]\end{array}\right\rangle$ \\
\hline$T_{3}$ & $\left.\begin{array}{l}\langle[0.65,0.70] \\
{[0.20,0.30]} \\
{[0.35,0.50]}\end{array}\right\rangle$ & $\left\langle\begin{array}{l}{[0.60,0.75]} \\
{[0.20,0.30]} \\
{[0.25,0.35]}\end{array}\right\rangle$ & $\left\langle\begin{array}{l}{[0.70,0.80],} \\
{[0.20,0.25]} \\
{[0.30,0.35]}\end{array}\right\rangle$ & $\left\langle\begin{array}{l}{[0.65,0.75]} \\
{[0.30,0.35]} \\
{[0.35,0.45]}\end{array}\right\rangle$ & $\left.\begin{array}{l}([0.75,0.80], \\
{[0.20,0.30],} \\
{[0.15,0.25]}\end{array}\right\rangle$ \\
\hline$T_{4}$ & $\left.\begin{array}{l}\langle[0.50,0.55] \\
{[0.25,0.35]} \\
{[0.40,0.45]}\end{array}\right\rangle$ & $\left\langle\begin{array}{l}{[0.65,0.75],} \\
{[0.25,0.35]} \\
{[0.20,0.25]}\end{array}\right\rangle$ & $\left\langle\begin{array}{l}{[0.60,0.70],} \\
{[0.25,0.35]} \\
{[0.35,0.40]}\end{array}\right\rangle$ & $\left\langle\begin{array}{l}{[0.70,0.75],} \\
{[0.25,0.35]} \\
{[0.35,0.40]}\end{array}\right\rangle$ & $\begin{array}{l}\langle[0.50,0.60] \\
{[0.35,0.45]} \\
{[0.35,0.45]}\end{array}$ \\
\hline
\end{tabular}

Table 5. Standardized decision matrix $\tilde{G}^{(2)}$ given by the expert $D_{2}$

\begin{tabular}{|c|c|c|c|c|c|}
\hline & $B_{1}$ & $B_{2}$ & $B_{3}$ & $B_{4}$ & $B_{5}$ \\
\hline$T_{1}$ & $\left\langle\begin{array}{l}{[0.45,0.55],} \\
{[0.25,0.30]} \\
{[0.35,0.45]}\end{array}\right\rangle$ & $\left\langle\begin{array}{l}{[0.65,0.75],} \\
{[0.20,0.25]} \\
{[0.25,0.35]}\end{array}\right\rangle$ & $\left\langle\begin{array}{l}{[0.65,0.70],} \\
{[0.30,0.35],} \\
{[0.35,0.45]}\end{array}\right\rangle$ & $\left\langle\begin{array}{l}{[0.60,0.70],} \\
{[0.25,0.40]} \\
{[0.40,0.45]}\end{array}\right\rangle$ & $\left\langle\begin{array}{l}{[0.50,0.65],} \\
{[0.35,0.40]} \\
{[0.30,0.35]}\end{array}\right\rangle$ \\
\hline$T_{2}$ & $\left\langle\begin{array}{l}{[0.70,0.85]} \\
{[0.10,0.15]} \\
{[0.15,0.25]}\end{array}\right\rangle$ & $\left\langle\begin{array}{l}{[0.70,0.75],} \\
{[0.20,0.25]} \\
{[0.25,0.35]}\end{array}\right\rangle$ & $\left\langle\begin{array}{l}{[0.70,0.80],} \\
{[0.20,0.30]} \\
{[0.30,0.35]}\end{array}\right\rangle$ & $\left\langle\begin{array}{l}{[0.75,0.85],} \\
{[0.20,0.30]} \\
{[0.30,0.35]}\end{array}\right\rangle$ & $\left\langle\begin{array}{l}{[0.75,0.85],} \\
{[0.20,0.25]} \\
{[0.15,0.20]}\end{array}\right\rangle$ \\
\hline$T_{3}$ & $\left\langle\begin{array}{c}{[0.65,0.70],} \\
{[0.20,0.30],} \\
{[0.35,0.50]}\end{array}\right\rangle$ & $\left\langle\begin{array}{l}{[0.65,0.70],} \\
{[0.20,0.30]} \\
{[0.30,0.35]}\end{array}\right\rangle$ & $\left\langle\begin{array}{l}{[0.70,0.80],} \\
{[0.15,0.25],} \\
{[0.25,0.30]}\end{array}\right\rangle$ & $\left\langle\begin{array}{l}{[0.75,0.80],} \\
{[0.25,0.30],} \\
{[0.35,0.40]}\end{array}\right\rangle$ & $\left\langle\begin{array}{l}{[0.65,0.75],} \\
{[0.30,0.35],} \\
{[0.20,0.30]}\end{array}\right\rangle$ \\
\hline$T_{4}$ & $\left\langle\begin{array}{l}{[0.50,0.55],} \\
{[0.25,0.35],} \\
{[0.40,0.45]}\end{array}\right\rangle$ & $\left\langle\begin{array}{l}{[0.60,0.65],} \\
{[0.25,0.30]} \\
{[0.30,0.40]}\end{array}\right\rangle$ & $\left\langle\begin{array}{l}{[0.65,0.75],} \\
{[0.20,0.25]} \\
{[0.30,0.40]}\end{array}\right\rangle$ & $\left\langle\begin{array}{l}{[0.65,0.75],} \\
{[0.30,0.35],} \\
{[0.40,0.50]}\end{array}\right\rangle$ & $\left\langle\begin{array}{l}{[0.55,0.65],} \\
{[0.25,0.35],} \\
{[0.30,0.35]}\end{array}\right\rangle$ \\
\hline
\end{tabular}


Table 6. Standardized decision matrix $\tilde{G}^{(3)}$ given by the expert $D_{3}$

\begin{tabular}{|c|c|c|c|c|c|}
\hline & $B_{1}$ & $B_{2}$ & $B_{3}$ & $B_{4}$ & $B_{5}$ \\
\hline$T_{1}$ & $\left\langle\begin{array}{l}{[0.45,0.55],} \\
{[0.25,0.30]} \\
{[0.35,0.45]}\end{array}\right\rangle$ & $\left.\begin{array}{l}\langle[0.75,0.80] \\
{[0.15,0.20]} \\
{[0.20,0.25]}\end{array}\right\rangle$ & $\left\langle\begin{array}{l}{[0.65,0.75]} \\
{[0.30,0.40]} \\
{[0.40,0.45]}\end{array}\right\rangle$ & $\left\langle\begin{array}{l}{[0.65,0.75],} \\
{[0.30,0.35]} \\
{[0.35,0.40]}\end{array}\right\rangle$ & $\begin{array}{l}{[0.60,0.65],} \\
{[0.30,0.35],} \\
{[0.25,0.35]}\end{array}$ \\
\hline$T_{2}$ & $\left\langle\begin{array}{l}{[0.70,0.85],} \\
{[0.10,0.15]} \\
{[0.15,0.25]}\end{array}\right\rangle$ & $\left\langle\begin{array}{l}{[0.60,0.70],} \\
{[0.30,0.35]} \\
{[0.35,0.45]}\end{array}\right\rangle$ & $\left\langle\begin{array}{l}{[0.70,0.80],} \\
{[0.25,0.30]} \\
{[0.30,0.40]}\end{array}\right\rangle$ & $\left\langle\begin{array}{l}{[0.70,0.85],} \\
{[0.20,0.30]} \\
{[0.30,0.35]}\end{array}\right\rangle$ & 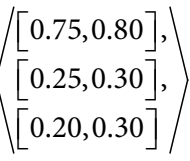 \\
\hline$T_{3}$ & $\left\langle\begin{array}{l}{[0.65,0.70],} \\
{[0.20,0.30]} \\
{[0.35,0.50]}\end{array}\right\rangle$ & $\left\langle\begin{array}{l}{[0.65,0.75],} \\
{[0.25,0.30]} \\
{[0.30,0.35]}\end{array}\right\rangle$ & $\left\langle\begin{array}{l}{[0.75,0.85],} \\
{[0.20,0.25]} \\
{[0.25,0.35]}\end{array}\right\rangle$ & $\left(\begin{array}{l}{[0.75,0.80]} \\
{[0.20,0.25]} \\
{[0.30,0.40]}\end{array}\right)$ & $\left.\begin{array}{l}{[0.80,0.85],} \\
{[0.10,0.20],} \\
{[0.10,0.20]}\end{array}\right\rangle$ \\
\hline$T_{4}$ & $\left\langle\begin{array}{l}{[0.50,0.55]} \\
{[0.25,0.35]} \\
{[0.40,0.45]}\end{array}\right\rangle$ & $\left\langle\begin{array}{l}{[0.70,0.75],} \\
{[0.20,0.30]} \\
{[0.25,0.35]}\end{array}\right\rangle$ & $\left\langle\begin{array}{l}{[0.60,0.65],} \\
{[0.35,0.45]} \\
{[0.45,0.50]}\end{array}\right\rangle$ & $\left\langle\begin{array}{l}{[0.60,0.70],} \\
{[0.35,0.40]} \\
{[0.40,0.50]}\end{array}\right\rangle$ & $\begin{array}{l}{[0.55,0.65],} \\
{[0.40,0.45]} \\
{[0.25,0.35]}\end{array}$ \\
\hline
\end{tabular}

Step 3. Integrate all decision matrices into the group decision matrix $\tilde{Q}=\left(\tilde{q}_{k o}\right)_{4 \times 5}$ by taking advantage of the Eq. (9) (see Table 7).

Table 7. Group decision matrix $\tilde{Q}$

\begin{tabular}{|c|c|c|c|c|c|}
\hline & $B_{1}$ & $B_{2}$ & $B_{3}$ & $B_{4}$ & $B_{5}$ \\
\hline$T_{1}$ & $\left\langle\begin{array}{l}{[0.4630,0.5945],} \\
{[0.2660,0.3318]} \\
{[0.3822,0.4827]}\end{array}\right\rangle$ & $\left(\begin{array}{l}{[0.7075,0.7885],} \\
{[0.1612,0.2115]} \\
{[0.2312,0.3111]}\end{array}\right)$ & $\left.\begin{array}{l}\langle[0.6130,0.7006], \\
{[0.2789,0.3448]} \\
\mid[0.3667,0.4694]\end{array}\right\rangle$ & $\left(\begin{array}{l}{[0.6381,0.7185]} \\
{[0.2866,0.3817]} \\
{[0.3817,0.4504]}\end{array}\right)$ & $\begin{array}{l}([0.5196,0.5963], \\
{[0.3498,0.4174]} \\
{[0.2815,0.3692]}\end{array}$ \\
\hline$T_{2}$ & $\left\langle\begin{array}{l}{[0.6709,0.7828],} \\
{[0.1516,0.2038]} \\
{[0.2038,0.2899]}\end{array}\right\rangle$ & $\left(\begin{array}{l}{[0.6471,0.7134],} \\
{[0.2345,0.2861]} \\
{[0.3025,0.3879]}\end{array}\right)$ & $\left.\begin{array}{l}\langle[0.7211,0.8217], \\
{[0.1927,0.2551]} \\
{[0.2551,0.3206]}\end{array}\right\rangle$ & $\left\langle\begin{array}{l}{[0.7134,0.8317]} \\
{[0.2187,0.2689]} \\
{[0.2815,0.3692]}\end{array}\right\rangle$ & $\left.\begin{array}{l}([0.7140,0.7811], \\
{[0.2543,0.3049]} \\
{[0.1861,0.2520]}\end{array}\right)$ \\
\hline$T_{3}$ & $\left\langle\begin{array}{l}{[0.7006,0.7648],} \\
{[0.1683,0.2487],} \\
{[0.2502,0.3516]}\end{array}\right\rangle$ & $\left(\begin{array}{l}{[0.6308,0.7383],} \\
{[0.2162,0.3166]} \\
{[0.2944,0.3667]}\end{array}\right)$ & $\begin{array}{l}\langle[0.7185,0.8192], \\
{[0.1861,0.2665]} \\
\mid[0.2866,0.3368]\end{array}$ & $\left\langle\begin{array}{l}{[0.7140,0.7813]} \\
{[0.2487,0.2994]} \\
{[0.3316,0.4193]}\end{array}\right\rangle$ & $\begin{array}{l}([0.7485,0.8088], \\
{[0.1737,0.2705],} \\
{[0.1399,0.2420]}\end{array}$ \\
\hline$T_{4}$ & $\left\langle\begin{array}{l}{[0.5270,0.5945],} \\
{[0.2420,0.3429],} \\
{[0.4031,0.4669]}\end{array}\right\rangle$ & $\begin{array}{l}{[0.6571,0.7281],} \\
{[0.2312,0.3191],} \\
{[0.2393,0.3163]}\end{array}$ & $\begin{array}{l}\mid[0.6131,0.6975], \\
{[0.2660,0.3513]} \\
\mid[0.3677,0.4325]\end{array}$ & $\left\langle\begin{array}{l}{[0.6552,0.7335],} \\
{[0.2944,0.3667]} \\
{[0.3792,0.4573]}\end{array}\right\rangle$ & $\left.\begin{array}{l}([0.5306,0.6308], \\
{[0.3372,0.4226]} \\
{[0.2994,0.3870]}\end{array}\right)$ \\
\hline
\end{tabular}


Step 4. Take advantage of the entropy weight method, the Eqs (10) and (11) to achieve original attribute weights $\imath_{1}=0.1854, l_{2}=0.2267, l_{3}=0.2034, \imath_{4}=0.1809, l_{5}=0.2036$ in line with the known information of the decision matrix $\tilde{Q}=\left(\tilde{q}_{k o}\right)_{4 \times 5}$.

Step 5. According to Eqs (12) and (13), compute the modified weights $\partial_{k i o}^{*}\left(\mathrm{t}_{o}\right)$ $(k, i=1,2, \cdots, h ; o=1,2, \cdots, p)$. The results are depicted in Table 8 to Table $11 .(a=0.61, b=$ 0.69 , based on the experiment of Kahneman (1992)).

Table 8 . The modified weights $\partial_{1 i o}^{*}\left(t_{o}\right)$

\begin{tabular}{|c|c|c|c|c|c|}
\hline & $B_{1}$ & $B_{2}$ & $B_{3}$ & $B_{4}$ & $B_{5}$ \\
\hline$\partial_{12}^{*}$ & 0.8868 & 1.0000 & 0.9368 & 0.8738 & 0.9373 \\
\hline$\partial_{13}^{*}$ & 0.8868 & 1.0000 & 0.9368 & 0.8738 & 0.9373 \\
\hline$\partial_{14}^{*}$ & 0.8868 & 1.0000 & 0.9368 & 0.8738 & 0.9373 \\
\hline
\end{tabular}

Table 10. The modified weights $\partial_{\text {kio }}^{*}\left(\mathrm{l}_{o}\right)$

\begin{tabular}{|c|c|c|c|c|c|}
\hline & $B_{1}$ & $B_{2}$ & $B_{3}$ & $B_{4}$ & $B_{5}$ \\
\hline$\partial_{31}^{*}$ & 0.9079 & 1.0000 & 0.9496 & 0.8971 & 0.9500 \\
\hline$\partial_{32}^{*}$ & 0.8868 & 1.0000 & 0.9368 & 0.8738 & 0.9491 \\
\hline$\partial_{34}^{*}$ & 0.9079 & 1.0000 & 0.9496 & 0.8971 & 0.9500 \\
\hline
\end{tabular}

Table 9. The modified weights $\partial_{2 i o}^{*}\left(t_{o}\right)$

\begin{tabular}{|c|c|c|c|c|c|}
\hline & $B_{1}$ & $B_{2}$ & $B_{3}$ & $B_{4}$ & $B_{5}$ \\
\hline$\partial_{21}^{*}$ & 0.9079 & 1.0000 & 0.9496 & 0.8971 & 0.9500 \\
\hline$\partial_{23}^{*}$ & 0.9079 & 1.0000 & 0.9496 & 0.8971 & 0.9382 \\
\hline$\partial_{24}^{*}$ & 0.9079 & 1.0000 & 0.9496 & 0.8971 & 0.9500 \\
\hline
\end{tabular}

Table 11. The modified weights $\partial_{\text {kio }}^{*}\left(\mathrm{t}_{o}\right)$

\begin{tabular}{|c|c|c|c|c|c|}
\hline & $B_{1}$ & $B_{2}$ & $B_{3}$ & $B_{4}$ & $B_{5}$ \\
\hline$\partial_{41}^{*}$ & 0.9079 & 1.0000 & 0.9496 & 0.8971 & 0.9500 \\
\hline$\partial_{42}^{*}$ & 0.8868 & 1.0000 & 0.9368 & 0.8738 & 0.9373 \\
\hline$\partial_{43}^{*}$ & 0.8868 & 1.0000 & 0.9368 & 0.8738 & 0.9373 \\
\hline
\end{tabular}

Step 6. Suppose $\eta=0.88, \mu=0.88$ and $\theta=2.25$ (Kahneman, 1992), compare the differences under each attribute between any two alternatives based on Eqs (14)-(15) (see Table 12). And then utilize Eq. (16) to put the holistic preponderance of alternative $T_{k}$ compared with alternative $T_{i}$ together. The relative predominance $\delta_{o}\left(T_{k}, T_{i}\right)$ and the overall predominance $\wp\left(T_{k}, T_{i}\right)$ are expressed in the relative predominance matrix $\delta_{o}=\left(\delta_{o}\left(T_{k}, T_{i}\right)\right)_{4 \times 4}(o=1,2,3$, $4,5)$ and the overall predominance matrix $\wp=\left(\wp\left(T_{k}, T_{i}\right)\right)_{4 \times 4}$.

Table 12. Distance between each of the two alternatives

\begin{tabular}{|c|c|c|c|c|c|}
\hline & $B_{1}$ & $B_{2}$ & $B_{3}$ & $B_{4}$ & $B_{5}$ \\
\hline$d_{o}\left(T_{1}, T_{2}\right)$ & 0.1683 & 0.0719 & 0.1109 & 0.0918 & 0.1333 \\
\hline$d_{o}\left(T_{1}, T_{3}\right)$ & 0.1420 & 0.0676 & 0.1013 & 0.0567 & 0.1722 \\
\hline$d_{o}\left(T_{1}, T_{4}\right)$ & 0.0227 & 0.0503 & 0.0101 & 0.0107 & 0.0165 \\
\hline$d_{o}\left(T_{2}, T_{3}\right)$ & 0.0362 & 0.0199 & 0.0118 & 0.0353 & 0.0389 \\
\hline$d_{o}\left(T_{2}, T_{4}\right)$ & 0.1563 & 0.0326 & 0.1044 & 0.0860 & 0.1304 \\
\hline$d_{o}\left(T_{3}, T_{4}\right)$ & 0.1300 & 0.0266 & 0.0948 & 0.0509 & 0.1693 \\
\hline
\end{tabular}




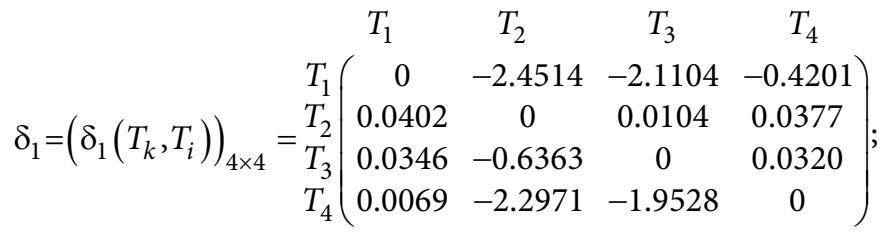

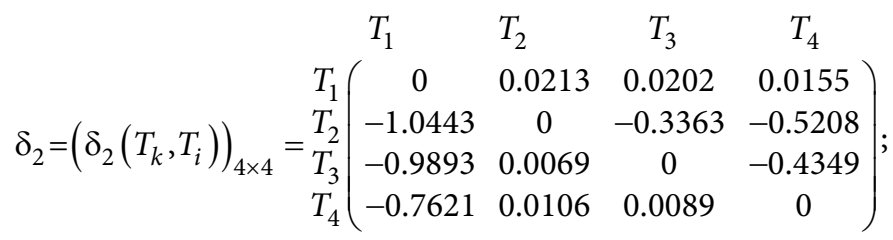

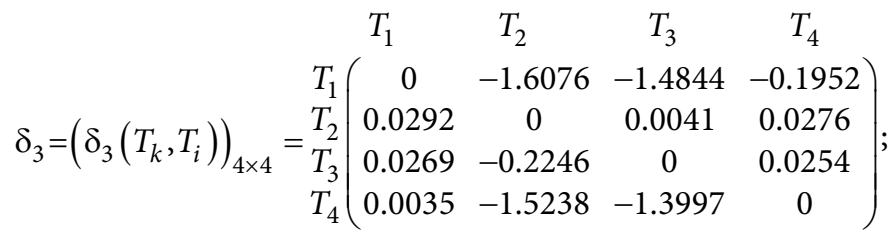

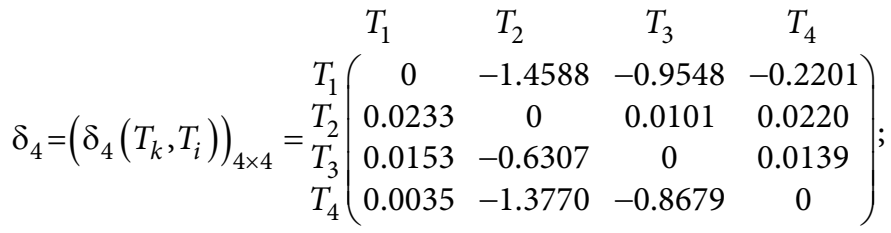

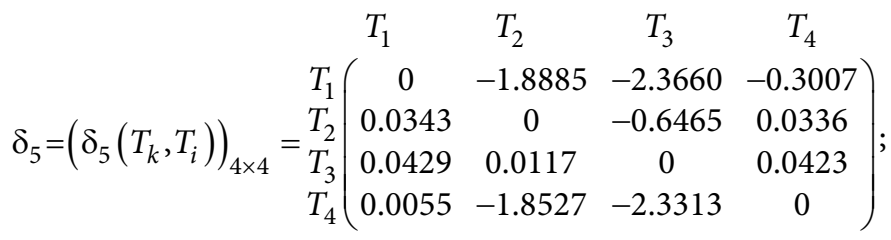

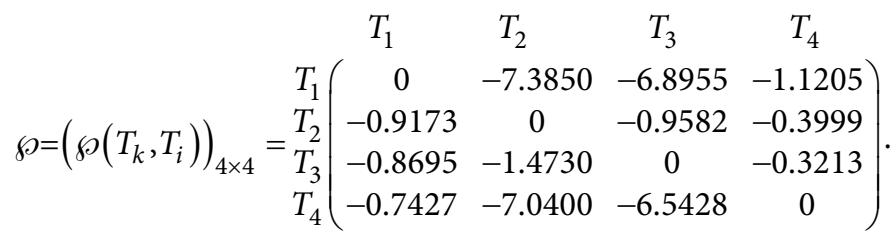

Step 7. In line with Eq. (19), standardize the overall predominance $\wp\left(T_{k}, T_{i}\right)$ to acquire the standardized overall predominance $\mathfrak{I}\left(T_{k}\right)(k=1,2,3,4)$ for the sake of comparison. The greater the standard overall predominance is, the better the alternative is.

$$
\begin{array}{r}
\mathfrak{I}\left(T_{1}\right)=0, \mathfrak{I}\left(T_{2}\right)=1, \mathfrak{I}\left(T_{3}\right)=0.9704, \mathfrak{I}\left(T_{4}\right)=0.0819 \\
T_{2}>T_{3}>T_{4}>T_{1} .
\end{array}
$$

Therefore, the alternative $T_{2}$ is the most excellent one. 


\section{Sensitivity analysis}

As parameters, the value of $a, b, \theta, \eta, \mu$ can be adjusted according to the needs of the actual situation and their value will affect the final calculation result to some extent. Therefore, in this section, we discuss the influence of variation of these parameters.

\subsection{The sensitivity analysis of parameters from the weighting function}

When the parameters $a$ and $b$ from the weighting function vary separately, we can plot the following graphs, Figure 1 and Figure 2, according to the values of the standardized overall predominance $\mathfrak{I}\left(T_{k}\right)(k=1,2,3,4)$. We can see clearly from the graphs that the ranking results remain the same in other cases except that the optimal alternative is changed when the value of parameter $b$ is 0.1 . However, changes in parameter $b$ have a slightly greater effect than changes in parameter $a$.

\subsection{The sensitivity analysis of parameters from the value function}

In this section, we also discuss the effects of individual changes in parameters $\theta, \eta$, and $\mu$ of the value function. The corresponding results are also presented in the form of graphs, Figure 3, Figure 4 and Figure 5. In general, the individual variation of the three parameters does not affect the ranking result of alternatives. However, the fluctuation of values of the standardized overall predominance $\mathfrak{I}\left(T_{k}\right)$ caused by the change of parameter $\mu$ is relatively larger.



Figure 1. When parameter $a$ changes alone, the results of $\mathfrak{I}\left(T_{k}\right)$ in different $a$



Figure 2. When parameter $b$ changes alone, the results of $\mathfrak{I}\left(T_{k}\right)$ in different $b$ 




Figure 3. When parameter $\theta$ changes alone, the results of $\mathfrak{I}\left(T_{k}\right)$ in different $\theta$



Figure 4. When parameter $\eta$ changes alone, the results of $\mathfrak{I}\left(T_{k}\right)$ in different $\eta$



Figure 5. When parameter $\mu$ changes alone, the results of $\mathfrak{I}\left(T_{k}\right)$ in different $\mu$

\section{Comparative analysis}

Comparing with other testified methods is indispensable for any new method which is to be proven effective. For verifying this new proposed CPT-IN-TODIM method, in this part, we choose Interval neutrosophic cross-entropy (Sahin, 2017), interval neutrosophic TODIM method (Xu et al., 2017), INWA operator and INWG operator (Garg \& Nancy, 2020) to compare with the new method in this paper. Table 13 exhibits the results from different selected methods. 
Table 13. The sequence from different methods

\begin{tabular}{|l|c|c|}
\hline \multicolumn{1}{|c|}{ Method } & The sequence & The best alternative \\
\hline Interval Neutrosophic Cross-entropy (Sahin, 2017) & $T_{2}>T_{3}>T_{1}>T_{4}$ & $T_{2}$ \\
\hline IN-TODIM method (Xu et al., 2017) & $T_{2}>T_{3}>T_{4}>T_{1}$ & $T_{2}$ \\
\hline INWA operator (Garg \& Nancy, 2020) & $T_{2}>T_{3}>T_{1}>T_{4}$ & $T_{2}$ \\
\hline INWG operator (Garg \& Nancy, 2020) & $T_{2}>T_{3}>T_{1}>T_{4}$ & $T_{2}$ \\
\hline CPT-IN-TODIM & $T_{2}>T_{3}>T_{4}>T_{1}$ & $T_{2}$ \\
\hline
\end{tabular}

On the basis of above comparison, there is no doubt that the alternative $T_{2}$ is the best one. In addition, each method has slightly different in aspects of results and concepts. Obviously, the CPT-IN-TODIM method has markedly superiority in describing decision maker's psychological states.

\section{Conclusions}

In this paper, after absorbing the experiences and deficiencies of previous studies, we establish the developed CPT-IN-TODIM method which is an expansion of MAGDM method with INSs. For one thing, this new developed CPT-IN-TODIM method, utilizing the weighting function to adjust attribute weights and the value function to improve the relative predominance, has marked superiority in describing decision maker's psychological states. For another, the new developed method not only takes a more scientific approach to acquire the original attribute weights, but also utilizes interval neutrosophic number as the tool to evaluate the uncertain information. Finally, the results of contrastive analysis indicates that this new developed method can lead to the appropriate conclusion and sticks out the differences between alternatives to provide clearer direction. Hence, the new developed CPT-IN-TODIM method is reliable and valid. In the future, we shall continue to explore the application of such method in other fields and put forward more scientific and reasonable new methods to solve MAGDM problems.

\section{References}

Awang, A., Aizam, N. A. H., Ab Ghani, A. T., Othman, M., \& Abdullah, L. (2020). A normalized weighted bonferroni mean aggregation operator considering shapley fuzzy measure under intervalvalued neutrosophic environment for decision-making. International Journal of Fuzzy Systems, 22, 321-336. https://doi.org/10.1007/s40815-019-00752-5

Biswas, P., Pramanik, S., \& Giri, B. C. (2016). TOPSIS method for multi-attribute group decision-making under single-valued neutrosophic environment. Neural Computing \& Applications, 27, 727-737. https://doi.org/10.1007/s00521-015-1891-2

Chen, S. M., Yang, M. W., Lee, L. W., \& Yang, S. W. (2012). Fuzzy multiple attributes group decisionmaking based on ranking interval type-2 fuzzy sets. Expert Systems with Applications, 39(5), 52955308. https://doi.org/10.1016/j.eswa.2011.11.008 
Duong, T. T. T., Phong, L. T., Hoi, L. Q., \& Thao, N. X. (2020). A novel model based on similarity measure and quality function deployment on interval neutrosophic sets for evaluation and selection market segments. Journal of Intelligent \& Fuzzy Systems, 38(4), 5203-5214. https://doi.org/10.3233/JIFS-191790

Fan, J. P., Li, D. D., \& Wu, M. Q. (2021). An extended TODIM method with unknown weight information under interval-valued neutrosophic environment for FMEA. International Journal of Computational Intelligence Systems, 14(1), 174-186. https://doi.org/10.2991/ijcis.d.201109.003

Flavio, A., \& GonzIlez, X. (2012). Behavioral multi-criteria decision analysis: Further elaborations on the todim method. Foundations of Computing Decision Sciences, 37(1), 3-8. https://doi.org/10.2478/v10209-011-0001-1

Garg, H., \& Nancy. (2020). Multiple attribute decision making based on immediate probabilities aggregation operators for single-valued and interval neutrosophic sets. Journal of Applied Mathematics and Computing, 63, 619-653. https://doi.org/10.1007/s12190-020-01332-9

Gomes, L. F. A. M., \& Lima, M. M. P. P. (1979). TODIM: Basics and application to multicriteria ranking of projects with environmental impacts. Foundations of Computing and Decision Sciences, 16, $113-127$.

Huang, Y., Lin, R., \& Chen, X. (2021). An enhancement EDAS method based on Prospect Theory. Technological and Economic Development of Economy, 27(5), 1019-1038. https://doi.org/10.3846/tede.2021.15038

Ji, X. N., Yu, L. X., \& Fu, J. P. (2020). Evaluating personal default risk in P2P lending platform: Based on dual hesitant Pythagorean fuzzy TODIM approach. Mathematics, $8(1), 8$. https://doi.org/10.3390/math8010008

Kahraman, C., Oztaysi, B., \& Onar, S. C. (2020). Single \& interval-valued neutrosophic AHP methods: Performance analysis of outsourcing law firms. Journal of Intelligent \& Fuzzy Systems, 38(1), 749-759. https://doi.org/10.3233/JIFS-179446

Kahneman, T. (1992). Advances in prospect theory: Cumulative representation of uncertainty. Journal of Risk and Uncertainty, 5, 297-323. https://doi.org/10.1007/BF00122574

Kahneman, D., \& Tversky, A. (1979). Prospect theory: An analysis of decision under risk. Econometrica, 47(2), 263-291. https://doi.org/10.2307/1914185

Keshavarz-Ghorabaee, M., Govindan, K., Amiri, M., Zavadskas, E. K., \& Antucheviciene, J. (2019). An integrated Type-2 fuzzy decision model based on WASPAS and SECA for evaluation of sustainable manufacturing strategies. Journal of Environmental Engineering and Landscape Management, 27(4), 187-200. https://doi.org/10.3846/jeelm.2019.11367

Kim, S. H., Choi, S. H., \& Kim, J. K. (1999). An interactive procedure for multiple attribute group decision making with incomplete information: Range-based approach. European Journal of Operational Research, 118(1), 139-152. https://doi.org/10.1016/S0377-2217(98)00309-9

Lei, F., Wei, G., \& Chen, X. (2021). Model-based evaluation for online shopping platform with probabilistic double hierarchy linguistic CODAS method. International Journal of Intelligent Systems, 36(9), 5339-5358. https://doi.org/10.1002/int.22514

Liang, Y. Y., Tu, Y., Ju, Y. B., \& Shen, W. J. (2019). A multi-granularity proportional hesitant fuzzy linguistic TODIM method and its application to emergency decision making. International Journal of Disaster Risk Reduction, 36, 101081. https://doi.org/10.1016/j.ijdrr.2019.101081

Liao, N., Wei, G., \& Chen, X. (2021). TODIM method based on cumulative prospect theory for multiple attributes group decision making under Probabilistic Hesitant Fuzzy setting. International Journal of Fuzzy Systems. https://doi.org/10.1007/s40815-021-01138-2

Liu, D. H., Liu, Y. Y., \& Wang, L. Z. (2019). Distance measure for Fermatean fuzzy linguistic term sets based on linguistic scale function: An illustration of the TODIM and TOPSIS methods. International Journal of Intelligent Systems, 34(11), 2807-2834. https://doi.org/10.1002/int.22162 
Liu, P. D., \& Teng, F. (2019). Probabilistic linguistic TODIM method for selecting products through online product reviews. Information Sciences, 485, 441-455. https://doi.org/10.1016/j.ins.2019.02.022

Meng, F. Y., Wang, N., \& Xu, Y. W. (2019). Interval neutrosophic preference relations and their application in virtual enterprise partner selection. Journal of Ambient Intelligence and Humanized Computing, 10, 5007-5036. https://doi.org/10.1007/s12652-019-01178-5

Nafei, A. H., Yuan, W. J., \& Nasseri, H. (2019). Group multi-attribute decision making based on interval neutrosophic sets. Studies in Informatics and Control, 28(3), 309-316. https://doi.org/10.24846/v28i3y201907

Nie, S., Liao, H. C., Wu, X. L., \& Xu, Z. S. (2019). Green supplier selection with a continuous intervalvalued linguistic TODIM method. IEEE Access, 7, 124315-124328. https://doi.org/10.1109/ACCESS.2019.2937994

Pramanik, S., Dalapati, S., Alam, S., \& Roy, T. K. (2016). TODIM method for group decision making under bipolar neutrosophic set environment. In F. Smarandache \& S. Pramanik (Eds.), New trends in neutrosophic theory and applications (vol. II, pp. 140-155). Infinite Study.

Pramanik, S., \& Mallick, R. (2019). TODIM strategy for multi-attribute group decision making in trapezoidal neutrosophic number environment. Complex \& Intelligent Systems, 5, 379-389. https://doi.org/10.1007/s40747-019-0110-7

Pramanik, S., Dalapati, S., Alam, S., \& Roy, T. K. (2017). NC-TODIM-based MAGDM under a neutrosophic cubic set environment. Information, 8(4), 149. https://doi.org/10.3390/info8040149

Pramanik, S., Dalapati, S., Alam, S., \& Roy, T. K. (2018). NC-VIKOR based MAGDM strategy under neutrosophic cubic set environment. Neutrosophic Sets and Systems, 20, 95-108. https://www. researchgate.net/publication/324844596_NC-VIKOR_Based_MAGDM_Strategy_under_Neutrosophic_Cubic_Set_Environment_Neutrosophic_Sets_and_Systems_vol_20_2018_pp_95-108

Sahin, R. (2017). Cross-entropy measure on interval neutrosophic sets and its applications in multicriteria decision making. Neural Computing \& Applications, 28, 1177-1187. https://doi.org/10.1007/s00521-015-2131-5

Smarandache, F. (2002). A unifying field in logics: Neutrosophic logic. Multiple-Valued Logic, 8, 13-19.

Su, Y., Zhao, M., Wei, C., \& Chen, X. (2021). PT-TODIM method for probabilistic linguistic MAGDM and application to industrial control system security supplier selection. International Journal of Fuzzy Systems. https://doi.org/10.1007/s40815-021-01125-7

Tang, G. (2016). Approaches for relational multiple attribute decision making with interval neutrosophic numbers based on Choquet Integral [Master Thesis]. Shandong University of Finance and Economics. Jinan, China.

Tian, X. L., Xu, Z. S., \& Gu, J. (2019). An extended TODIM based on cumulative prospect theory and its application in venture capital. Informatica, 30(2), 413-429.

https://doi.org/10.15388/Informatica.2019.212

Wang, H., Smarandache, F., Zhang, Y., \& Sunderraman, R. (2004). Interval neutrosophic logics: Theory and applications, 1-21. arXiv:cs/0410056v2

Wang, H., Smarandache, F., Zhang, Y. Q., \& Sunderraman, R. (2005). Interval neutrosophic sets and logic: Theory and applications in computing. Hexis. https://digitalrepository.unm.edu/math_fsp/38

Wang, H., Smarandache, F., Zhang, Y. Q., \& Sunderraman, R. (2010). Single valued neutrosophic sets. Multispace Multistruct, 10, 410-413. http://fs.unm.edu/SingleValuedNeutrosophicSets.pdf

Wang, J., Gao, H., \& Lu, M. (2019). Approaches to strategic supplier selection under interval neutrosophic environment. Journal of Intelligent \& Fuzzy Systems, 37(2), 1707-1730.

https://doi.org/10.3233/JIFS-179235 
Wang, T. J. (2020). The projection model with unknown weight information under interval neutrosophic environment and its application to software quality-in-use evaluation. Mathematical Problems in Engineering, 2020, 7279420. https://doi.org/10.1155/2020/7279420

Wei, G., Wei, C., Wu, J., \& Guo, Y. (2021). Probabilistic linguistic multiple attribute group decision making for location planning of electric vehicle charging stations based on the generalized Dice similarity measures. Artificial Intelligence Review, 54, 4137-4167. https://doi.org/10.1007/s10462-020-09950-2

Wu, H. B., Yuan, Y., Wei, L. J., \& Pei, L. D. (2018). On entropy, similarity measure and cross-entropy of single-valued neutrosophic sets and their application in multi-attribute decision making. Soft Computing, 22, 7367-7376. https://doi.org/10.1007/s00500-018-3073-5

Xu, D. S., Wei, C., \& Wei, G. W. (2017). TODIM method for single-valued neutrosophic multiple attribute decision making. Information, 8(4), 125. https://doi.org/10.3390/info8040125

Xu, Z. S., \& Chen, J. (2007). An interactive method for fuzzy multiple attribute group decision making. Information Sciences, 177(1), 248-263. https://doi.org/10.1016/j.ins.2006.03.001

Yang, L. H., Li, D. M., \& Tan, R. P. (2020). Research on the shortest path solution method of interval valued neutrosophic graphs based on the ant colony Algorithm. IEEE Access, 8, 88717-88728. https://doi.org/10.1109/ACCESS.2020.2990912

Zadeh, L. A. (1965). Fuzzy sets. Information and Control, 8(3), 338-353. https://doi.org/10.1016/S0019-9958(65)90241-X

Zhang, Y. X., Xu, Z. S., \& Liao, H. C. (2019). Water security evaluation based on the TODIM method with probabilistic linguistic term sets. Soft Computing, 23, 6215-6230. https://doi.org/10.1007/s00500-018-3276-9

Zhao, M., Wei, G., Chen, X., \& Wei, Y. (2021a). Intuitionistic fuzzy MABAC method based on cumulative prospect theory for multiple attribute group decision making. International Journal of Intelligent Systems, 36(11), 6337-6359. https://doi.org/10.1002/int.22552

Zhao, M., Wei, G., Guo, Y., \& Chen, X. (2021b). CPT-TODIM method for interval-valued bipolar fuzzy multiple attribute group decision making and application to industrial control security service provider selection. Technological and Economic Development of Economy, 27(5), 1186-1206. https://doi.org/10.3846/tede.2021.15044

Zhao, M. W., Wei, G. W., Wei, C., \& Wu, J. (2021c). Improved TODIM method for intuitionistic fuzzy MAGDM based on cumulative prospect theory and its application on stock investment selection. International Journal of Machine Learning and Cybernetics, 12, 891-901. https://doi.org/10.1007/s13042-020-01208-1

Zhao, M. W., Wei, G. W., Wei, C., Wu, J., \& Wei, Y. (2021d). Extended CPT-TODIM method for interval-valued intuitionistic fuzzy MAGDM and its application to urban ecological risk assessment. Journal of Intelligent \& Fuzzy Systems, 40(3), 4091-4106. https://doi.org/10.3233/JIFS-200534 